\title{
EDITORIALE
}

\section{COMPETIZIONE E SPECIFICITÀ DEL "MADE IN ITALY": NOTE IN MARGINE AL RUOLO DELLA NOSTRA DISCIPLINA}

\author{
Guido Cristini
}

\begin{abstract}
"Guardando al futuro bisogna far sì che il Paese possa riuscire a mantenere vitale e solida la sua vocazione manifatturiera, dove è radicato il cuore del Made in Italy"
\end{abstract}

Riccardo Varaldo ${ }^{1}$

Nel corso di questi ultimi anni la difesa della competitività del "Made in Italy" è risultata una tematica oggetto di particolare attenzione nel nostro Paese in ragione della sua crescente rilevanza. Se ci si limita a considerare l'ultimo anno sono una decina i libri pubblicati sull'argomento, nonché non meno di un centinaio gli articoli pubblicati e i convegni organizzati di cui si rintraccia ampia eco nei principali media nazionali.

In particolare, il quesito di fondo di tale dibattito rimanda a come "Made in Italy", ovvero i prodotti, le marche, i processi produttivi che connotano la tradizione manifatturiera del nostro Paese, possa mantenere anche in futuro un ruolo distintivo (e difendibile) in un mondo sempre più globalizzato.

In questo quadro, provo ad avanzare alcune brevi riflessioni relative al compito che noi studiosi di marketing possiamo svolgere per comprendere meglio la natura della sfida che ha di fronte il "Made in Italy" e per indicare le azioni più opportune che questo è chiamato ad intraprendere al fine di difendere la posizione competitiva.

Innanzitutto, forse ci si dimentica che quello definiamo "Made in Italy" è formato, in larga misura, da piccole e medie imprese. Dalla ricerca realizzata da Mediobanca-Unioncamere (2009) delle 4345 medie imprese censite in Italia, quelle riconducibili al "made in Italy" concentrano oltre il $62 \%$ del fatturato complessivo. Se si aggiungono anche le piccole imprese che, in buona parte, operano in contesti settoriali tradizionali dove è prevalente il riferimento all'italianità dei prodotti, appare evidente come questo segmento di imprese risulti uno degli snodi fondamentali per il rilancio economico del nostro Paese.

1. Considerazione tratta dall'editoriale: Le medie imprese: una risposta alla crisi, Mercati \& Competitività, 3, 2010.

Mercati e Competitività n. 3, 2011 


\section{G. Cristini}

$\mathrm{Nel}$ dibattito recente su cosa sia strategico e prioritario in Italia ${ }^{2}$, diversi autori si sono interrogati su quali settori sia opportuno investire, e, in tale ambito, quali tipologie di intervento risultino prioritarie a livello di sistema.

Si tratta di comprendere se sia essenziale, o meno, progettare interventi specifici a favore dei principali settori del manifatturiero italiano riconducibili al core della nostra offerta "Made in Italy" e costituiti dal "Quadrilatero delle quattro A" dell'eccellenza italiana, della tradizione, del design. Parliamo, per intenderci, di arredamento, abbigliamento, alimentare e apparecchiatura industriale, settori nei quali, ancor oggi, il nostro Paese è riconosciuto ed apprezzato a livello mondiale.

Macro settori che, ovviamente, non sono strategici nell'accezione classica del termine come lo possono essere l'energia, la difesa o le telecomunicazioni, ma nei quali, tuttavia, il nostro Paese detiene una leadership che è, al tempo stesso, economica e culturale, retaggio di una lunga e consolidata tradizione manifatturiera. Settori che, forse, se non supportati da politiche economiche mirate, rischiano un declino lento, ma inarrestabile.

In questi settori, l'Italia ha rappresentato da sempre un riferimento costante per i diversi operatori presenti a livello mondiale in tema di design, di qualità, di funzionalità. In tali ambiti, il sapere deriva da una storia di conoscenze, di capacità presenti in territori o in distretti nei quali la cultura di quel prodotto o di quel processo hanno fatto sì che negli altri Paesi quel determinato comparto fosse sinonimo di "eccellenza italiana". Piace a chi scrive la definizione di "Made in Italy" proposta di recente da un imprenditore di una piccola impresa italiana e riportata in un testo appena pubblicato (Romano, 2011), come "la capacità di privilegiare il lungo termine sul breve termine. La lealtà verso il mercato contro la mistificazione del prodotto finalizzata a produrre una crescita vertiginosa e spesso ingiustificata.... Ė passione e rispetto della tradizione. È sedurre l'economia facendo le cose che facevano gli altri prima di noi e rassicurarla facendo quel qualcosa sempre un po' meglio..."3.

Ecco, è in questo miglioramento incrementale, continuo, che la tradizione diventa portato culturale, costituisce sapere che viene trasmesso attraverso le generazioni. Rappresentare e difendere la tradizione significa per molte Pmi motivo di orgoglio in quanto contribuisce a creare valore per tutti coloro che "abitano" nel territorio (reale o figurato che sia), nel quale

2. Di recente sulla stampa nazionale è stato dibattuto circa l'opportunità di mantenere italiana la Parmalat di fronte all'acquisizione di azioni da parte di Lactalis. Attualmente l'impresa francese ha lanciato un'Opa sull'intera base azionaria di cui al momento (l'editoriale è stato redatto in data 20.5.2011) non si conosce l'esito.

3. Da Passione Italia, pag. 42 di Fabiana Romano, Mondadori, 2011. Si tratta della parte finale dell'intervista realizzata con Fabrizio Torrini, titolare dell'azienda orafa famigliare più antica del mondo. 
è cresciuta l'impresa. Dentro il distretto, all'interno di una area geografica, nei diversi stadi della filiera, in un sistema di relazioni fondato, al tempo stesso, sullo scambio di informazioni e di interessi.

In questa prospettiva, assume rilevanza il ruolo delle relazioni di lunga durata, della natura incrementale dello scambio con altri attori, delle dimensioni fiduciarie che si generano, come, peraltro, alcune teorie di management hanno già da tempo evidenziato ${ }^{4}$.

Dal pregevole lavoro realizzato in ambito SIM da diversi colleghi (Dalli, Resciniti, Tunisini, 2010) ${ }^{5}$ nel biennio 2008-2009 e incentrato sulle Medie imprese, sono riportati in modo esaustivo alcuni fattori distintivi che connotano le condotte delle imprese del "Made in Italy". Tra questi, tre appaiono a chi scrive, di particolare rilevanza nell'attuale scenario di mercato. Innanzitutto, il tema delle crescita dimensionale e della sfida internazionale. In secondo luogo, la configurazione delle relazioni extra-organizzative, ovvero il potenziamento del modello a rete. Infine, il modello di imprenditorialità famigliare e il passaggio generazionale.

\section{Crescita dimensionale}

Un primo importante fattore che viene sollevato in tema di competitività dell'eccellenza italiana rimanda alla dimensione di impresa e agli ostacoli/problemi che le Pmi si trovano ad affrontare per aumentare la scala, in presenza di un crescente processo di globalizzazione dei mercati ${ }^{6}$. In altri termini, possono le Pmi difendere la loro posizione competitiva nel tempo e, attraverso il potenziamento dei fattori distintivi della marca, difendere positivamente gli elementi di differenziazione che hanno saputo creare nel passato? Ed ancora, il tragitto per la crescita che si va a prefigurare è unico, ovvero segue una traiettoria uniforme, una sorta di binario obbligato, $\mathrm{o}$, al contrario, è possibile prevedere "differenze", "specificità", scarti"? Percorsi, per intenderci, fondati su sistemi organizzativi propri, su culture specifiche, su best practices non riconducibili in modo automatico al riferimento unico dell' "one best way"?

I casi di successo studiati sembrano confermare la tesi che lo sviluppo dimensionale, nonchè la capacità di affermazione nei mercati internazionali da parte delle Pmi siano da ricondursi a processi di crescita difformi nei

4. Si fa riferimento in particolare al filone riguardante la "Resource-based Theory". Cfr. Wernerfelt (1984), The Resource-Based View of the Firm. Strategic Management Journal, $5,(2)$.

5. Il gruppo di lavoro sulla media impresa cui fa riferimento l'articolo citato contava oltre 60 ricercatori articolati il 29 gruppi di ricerca.

6. Si rimanda anche su questo aspetto al lavoro prima citato di Dalli, Resciniti e Tunisini (2010) pubblicato sulla nostra Rivista. 


\section{G. Cristini}

quali "le strategie di focalizzazione dinamica sono realizzate secondo percorsi "firm-specific" che fanno leva... su tre fattori: l'orientamento all'innovazione,la personalizzazione del prodotto, il presidio del mercato..." Elementi che, con diversa intensità, appaiono i drivers di sviluppo delle imprese del "Made in Italy", anche in quei comparti dove il valore è fondato sulla tradizione e sulla qualità dei prodotti (si pensi all'alimentare, ad esempio). Le tradizionali competenze di matrice produttiva si evolvono "poiché gli intangibles sono determinati per affermarsi in ambiti concorrenziali dove il vantaggio competitivo si afferma oltre la fabbrica"8.

Secondo Preti, (2011) che ha analizzato le determinanti di creazione del valore da parte delle imprese minori, poi, il successo di una parte non marginale delle Pmi italiane, deriva proprio dall'originalità delle scelte realizzate in chiave strategico- organizzativa, condotte attraverso il perseguimento di percorsi propri, difficilmente codificabili all'interno del paradigma del modello manageriale di matrice nordamericana9 .

La generazione di valore è, secondo questa prospettiva, in larga misura, da correlarsi alla capacità che ogni impresa manifesta nel produrre sapere specifico, che contraddistingue e qualifica il settore, la filiera, il distretto o la rete nella quale opera. Non esiste un solo modello al quale riferirsi per migliorare la propria posizione competitiva, ma più modelli, più strategie, più traiettorie da percorrere a seconda delle caratteristiche distintive di natura organizzativa, culturale e conoscitiva che connotano ciascuna impresa all'interno del proprio ambito competitivo. Per spiegare i successi ottenuti da alcune imprese minori nel corso di questi anni, in particolare quelle che sono state capaci di reagire alla crisi e, alla fine di un processo di riorganizzazione, di uscirne rafforzate, è improprio e, forse sbagliato, individuare un unico riferimento, una sorta di ricetta valida per tutti e tutto.

\section{Potenziamento della rete di relazioni}

Un secondo fattore che caratterizza le Pmi e contribuisce a spiegare la loro condotta è costituito dall'operare all'interno di un sistema a rete. Le imprese minori sono, per loro natura, chiamate a far leva sull'interdipendenza positiva con altri attori presenti nell'ambiente competitivo (filiera, distretto, settore) al fine di migliorare la posizione competitiva. Come ricorda Rullani nel testo appena citato (Preti, 2011), la possibilità di crescere di tali imprese dipende dalla consapevolezza di operare in un sistema costituito spesso da tanti altri operatori. È necessario, ci ricorda, che nelle

7. Ibidem.

8. Ibidem.

9. Si rimanda a P. Preti, Il meglio del piccolo, Egea, 2011. 
Pmi coesista la dimensione individuale, soggettiva, con quella collettiva, la quale deriva dalla consapevolezza di operare in molti casi interagendo all'interno di un contesto più ampio del sistema ${ }^{10}$. L'impresa minore ha successo, in molti casi, nella misura in cui comprende di essere una parte dell'ambiente competitivo nel quale ha interessi e relazioni. Si pensi, a tale fine, all'importanza dell'immagine collettiva per il successo di un determinato settore, segmento, tipologia di prodotto al cospetto di una domanda finale internazionale.

In tale prospettiva, è centrale che gli studiosi di marketing contribuiscano a fornire indicazioni utili al sistema delle Pmi italiane, mediante modelli e strumenti specifici, in grado di meglio individuare le determinanti del successo competitivo all'interno del singolo mercato (o della singola filiera). Perseguendo un approccio che tenga conto che parte del successo ottenuto da tali imprese può derivare dal corretto mix tra elementi di natura competitiva e di natura collaborativa, ma anche recuperando solide competenze sulla struttura e il comportamento di che connota ogni settore (o filiera). In tal modo, contribuendo ad accrescere la consapevolezza delle stesse imprese sul fatto che il vantaggio competitivo si ottiene in diversi contesti settoriali anche dall'adozione di azioni di natura collaborativa fondate sullo scambio e sulla cooperazione con i rivali.

Nei settori "dell'eccellenza italiana" poi, da diverse parti ci si domanda se non sia giunto il tempo di ricorrere ad interventi di politica industriale, in grado di supportare il sistema delle imprese italiane attualmente chiamate a sostenere la competizione sui mercati internazionali, sfida di assoluta complessità quando si dispone di una scala dimensionale medio-piccola ${ }^{11}$.

Una politica che provi a raccordare, qualificare, sostenere le imprese di minori dimensioni e che, proprio attraverso un puntuale supporto in termini di conoscenze (informazioni, strumenti, processi, relazioni, etc.), sia in grado di fornire un concreto e duraturo contributo alla crescita.

\section{Governance famigliare e passaggio generazionale}

Un terzo fattore che contraddistingue la condotta delle Pmi operanti nei settori fortemente evocativi dell' 'italianità", riguarda la modalità ed i siste-

10. "il modello di comportamento che si ricava dallo studio degli stormi di uccelli... Ci offre un punto di vista utile per capire come l'individualismo soggettivo possa, nel nostro coesistere con una forte interdipendenza oggettiva... Ogni uccello che vola in una formazione a stormo, non ha in effetti consapevolezza della forma collettiva e della traiettoria seguita dall'insieme, ma si regola sulla base della posizione e direzione degli altri uccelli che gli volano vicino...".

11. In questi ultimi mesi sono diversi gli interventi promossi da Dario Di Vico sul Corriere della Sera, ai quali opportunamente si rimanda. 


\section{G. Cristini}

mi di governance dell'impresa, in larga misura, riconducibili alla capacità di esprimere valore attraverso un modello "di imprenditorialità famigliare".

Come è noto, un primo problema è rappresentato proprio dalla criticità ricoperta dalla trasmissione generazionale, ovvero dalle scelte che vengono effettuate per facilitare il passaggio delle responsabilità imprenditoriali all'interno della famiglia. A ben vedere la storia stessa dell'impresa in Italia è fortemente intrecciata con la vicenda imprenditoriale di molte famiglie, sul loro evolversi nel tempo, sulla capacità delle precedenti generazioni di trasmettere conoscenze, tradizioni, saperi a quelle nuove.

Tale fenomeno rimanda nell'immediato ad un quesito: come si può assicurare, secondo un modello di tipo "darwiniano", che con il passare delle generazioni, l'impresa non solo non perda i plus distintivi che la connotano, ma, al contrario, si rafforzi, rendendo tali specificità più forti e più ancorate al presente?

Il quesito appena richiamato sembra rimandare, in prevalenza ad aspetti di natura organizzativa ed istituzionale. In realtà, se si fa riferimento alla cultura prevalente e ai modelli di leadership adottati dall'impresa, appare evidente come questi discendano, in primo luogo, dalla vision imprenditoriale e dall'imprinting dettato dalla storia di quella famiglia.

Esistono sicuramente dei codici di relazione che si fondano sui valori espressi e condivisi all'interno della comunità famigliare che supportano, in questo senso, la scelta dei nuovi imprenditori. Si pensi, ad esempio, a fattori quali il merito, che vengono utilizzati nel processo di selezione all'interno della famiglia ogni qualvolta si presenti il problema del passaggio generazionale. In particolare, il meccanismo attraverso il quale si identifica il nuovo capo azienda nell'ambito di gruppo famigliare rappresenta, senza dubbio, una scelta determinante per assicurare il mantenimento del bene collettivo, costituito dall'impresa e dalle relazioni positive intessute con il "territorio".

Il processo di selezione e formazione dei nuovi imprenditori appare a chi scrive una delle determinanti del successo delle imprese famigliari, specialmente quelle che operano sui mercati internazionali e hanno il compito di comunicare i valori del "Made in Italy".

Nel leggere la storia dei diversi casi di imprese dell' "eccellenza italiana" certo è, che una parte importante dei successi ottenuti non possa che essere ricondotto al ruolo centrale delle risorse umane, capaci di essere il fulcro (e il motore) di una sintesi tra disegno strategico e azione operativa.

Sorge il dubbio che in un quadro come quello appena definito, basato sulle specificità dei saperi in relazione agli ambiti competitivi nei quali si opera ai modelli di impresa "industry specific", si perda la capacità di cogliere le vere determinanti del successo di quell'impresa e di quel nucleo famigliare.

Un successo che deriva, in grande misura, dalle scelte adottate in chiave competitiva dall'imprenditore, a loro volta, frutto della capacità di gestire 
processi diversi, trasversali, multi-funzionali. $\mathrm{O}$ ancora, al diverso ruolo assunto da determinati valori che sono alla base del processo decisionale. $\mathrm{Si}$ pensi in tale ambito, ad esempio, all'importanza riconosciuta alle dimensioni fiduciarie nelle relazioni tra proprietà e management, diversamente, ad esempio, da quanto avviene nelle imprese di grandi dimensioni, dove le determinanti di selezione e di sviluppo dei managers risultano fondate su altre variabili (competenze, capacità, assunzione del rischio, problem solving, etc.)

\section{Quesito (e dubbio) finale}

Nel tentativo di offrire qualche spunto di riflessione ed eventualmente concorrere alla discussione sul ruolo che può svolgere la nostra Associazione nel supportare le Pmi del "Made in Italy" nel processo di competizione globale, ritengo necessario, infine, avanzare un piccolo quesito.

È utile per la nostra economia (e società) disporre di ricercatori che, dato il percorso mono (o micro) disciplinare che devono percorrere per affermarsi, hanno sempre più difficoltà a fornire un contributo di conoscenza avanzata (in quanto specifica) alle imprese e alle reti che connotano e qualificano il nostro sistema Paese?

Non si dovrebbe, forse, suggerire un percorso diverso nel quale, proprio in relazione alla presenza di un unico paradigma di riferimento, sia in modo consapevole favorita l'adozione di approcci in grado di leggere meglio le specificità (longitudinali e latitudinali) che contraddistinguono quell'ampio spettro di settori, di distretti, di filiere nel quale si articola l'eccellenza italiana?

Senza questo piccolo processo di ri-orientamento che richiede l'adozione di una visione "trasversale" dei fenomeni che qualificano il processo di management, contestualizzata allo specifico modello di impresa, c'è il fondato rischio che una buona parte degli studi di marketing possa, nel complesso, risultare "poco utile" all'economia e alla società italiana.

L'idea di una forte internazionalizzazione dei saperi, alla quale, peraltro, molti di noi credono, disancorata dal contesto Paese nel quale si opera, non rischia, tuttavia, di contribuire a rendere poco fruttuoso il nostro fare ricerca?

Il dubbio si insinua quando si scopre che una parte non marginale dei casi di successo oggetto di studio nelle più prestigiose Business School americane raccontano delle imprese e dei brand italiani che operano in alcuni settori del "Made in Italy". Così come ogni anno ci viene segnalato che alcune iniziative post laurea realizzate nel nostro Paese in tema di design e di fashion sono costantemente prese d'assalto da studenti provenienti da tutto il mondo. 


\section{G. Cristini}

Non sarebbe più utile contribuire a studiare e supportare questo permanente miracolo economico fondato sull' "italian way"?

Si ha, per certi versi, l'impressione che, limitatamente a questo ambito economico, quello dell'eccellenza italiana, sia venuto il tempo di riconsiderare una prospettiva più "contingency", maggiormente attenta ai fattori distintivi, specifici, qualificanti.

Senza tale visione, noi accademici di marketing rischiamo di entrare, nostro malgrado, in due principali categorie. O quella degli "iper-specializzati", per la quale l'expertise deriva prevalentemente dallo studiare e approfondire un unico fenomeno/aspetto/problema, ciò che produce la prospettica incapacità a raccordare elementi, fatti, informazioni, tradendo, in tal modo, il messaggio a noi trasmesso dagli studi aziendalistici italiani. $\mathrm{O}$ c'è il pericolo di far parte della categoria dei propagatori di culture "altre", il cui valore principale è rappresentato spesso dal costante ricorso (a volte, acritico) alla strumentazione di natura quantitativa. Docenti di modelli generalisti, necessari per spiegare tutto, ma, al contempo, inadeguati ad interpretare i segnali deboli e immateriali dello specifico, territorio, filiera, distretto, comparto, impresa che sia.

Ciò che comporta quale immediata conseguenza il fatto di essere inadeguati a supportare la competitività che si fonderà in futuro anche sui fattori differenzianti del "manufacturing" italiano, quale portato di cultura, arte, tradizione, qualità.

Se ci dimentichiamo di questo, c'è il fondato pericolo di diventare degli onesti accademici chiamati ad imitare e ad adattare al nostro campo di osservazione un modello generalista, necessariamente onnicomprensivo, per certi versi "alto".

Ricercatori, insomma, che seguono acriticamente quanto ci viene trasmesso da altri, secondo un modello culturale che tende all'indifferenziazione, alla generalizzazione, all'omogeneizzazione. Insomma, per dirla nel nostro linguaggio, dei docenti imitatori, sostanzialmente dei "me too". 